\title{
Development of the Operando Atom Probe: The Influence of the electric field on Fe oxidation
}

Sten Lambeets ${ }^{1}$, Mark Wirth $^{2}$, Arun Devaraj $^{3}$ and Daniel Perea ${ }^{4}$

${ }^{1}$ Pacific Northwest National Laboratory, Washington, United States, ${ }^{2}$ Pacific Northwest National Laboratory (PNNL), Richland, Washington, United States, ${ }^{3}$ PNNL, United States, ${ }^{4}$ EMSL at PNNL, United States

Heterogeneous catalysis is one of the pillars of the chemical industry and the "green chemistry" concept. However, the sparse understanding of surface mechanisms at molecular scale impedes development of high-performance catalysts and requires development of new tools and methodologies. Heterogeneous catalysis is a surface phenomenon and in order to maximize the active surface available for a minimum of bulk material used, catalyst are presented as supported nanoparticles. Nanoparticle surfaces are the site of reaction processes and their size/shape drastically influence the catalyst's features and its performances towards the reaction of interest. Oxidation and corrosion of metals is another research field with very similar research questions. Metals used in variety of structural applications from nuclear reactor internals to automobiles degrade due to oxidation and corrosion. Both oxidation and corrosion are also fundamentally surface reaction mechanisms, with clear parallels to the heterogenous catalysis reaction mechanisms.

Starting from very sharp needles, whose needle apexes are similar to model nanoparticles by their sizes and shapes, we are able to explore these surface mechanisms at the nanoscale with the use of Atom Probe Microscopy (APM) techniques. From Field Emission Microscopy to Atom Probe Tomography, APM techniques were extensively used from the 1980s to track and identify reactions mechanisms. Our most recent development led us to adopt the approach introduced by Block et al. with their Pulsed Field Desorption Mass Spectrometry (PFDMS), using the electric field pulses frequencies applied in atom probe techniques to explore the surface dynamics.

In this work we dedicated our efforts to understand the electric field driven oxidation of Fe and Fe-alloys. A clean Fe needle sample is exposed at $2.5 \times 10^{-9}$ mbar of pure oxygen while held under an applied electric field inside the APT analysis chamber and the change in surface composition was measured as a function of time by running the atom probe at $302.9 \mathrm{~K}$ specimen temperature. The voltage pulse intensity is kept constant while the DC standing voltage is modified progressively to observe the impact of the latter on the oxide formation. The figure 1 shows the schematic of the operando APT set up. From the experiments we directly observed that the $\mathrm{V}_{\text {stat }}$ drastically changed the oxide composition measured in APT. For higher $\mathrm{V}_{\text {stat }}$, a relatively pure Fe surface was observed. on changing the $\mathrm{V}_{\text {stat }} \mathrm{a}$ direct change in the oxide stoichiometry was measured, establishing a powerful method to quantitatively study the electric field assisted oxidation of Fe. This approach can now be used for Fe based metallic alloys to reveal the oxidation mechanisms under the applied electric field conditions [1]. 


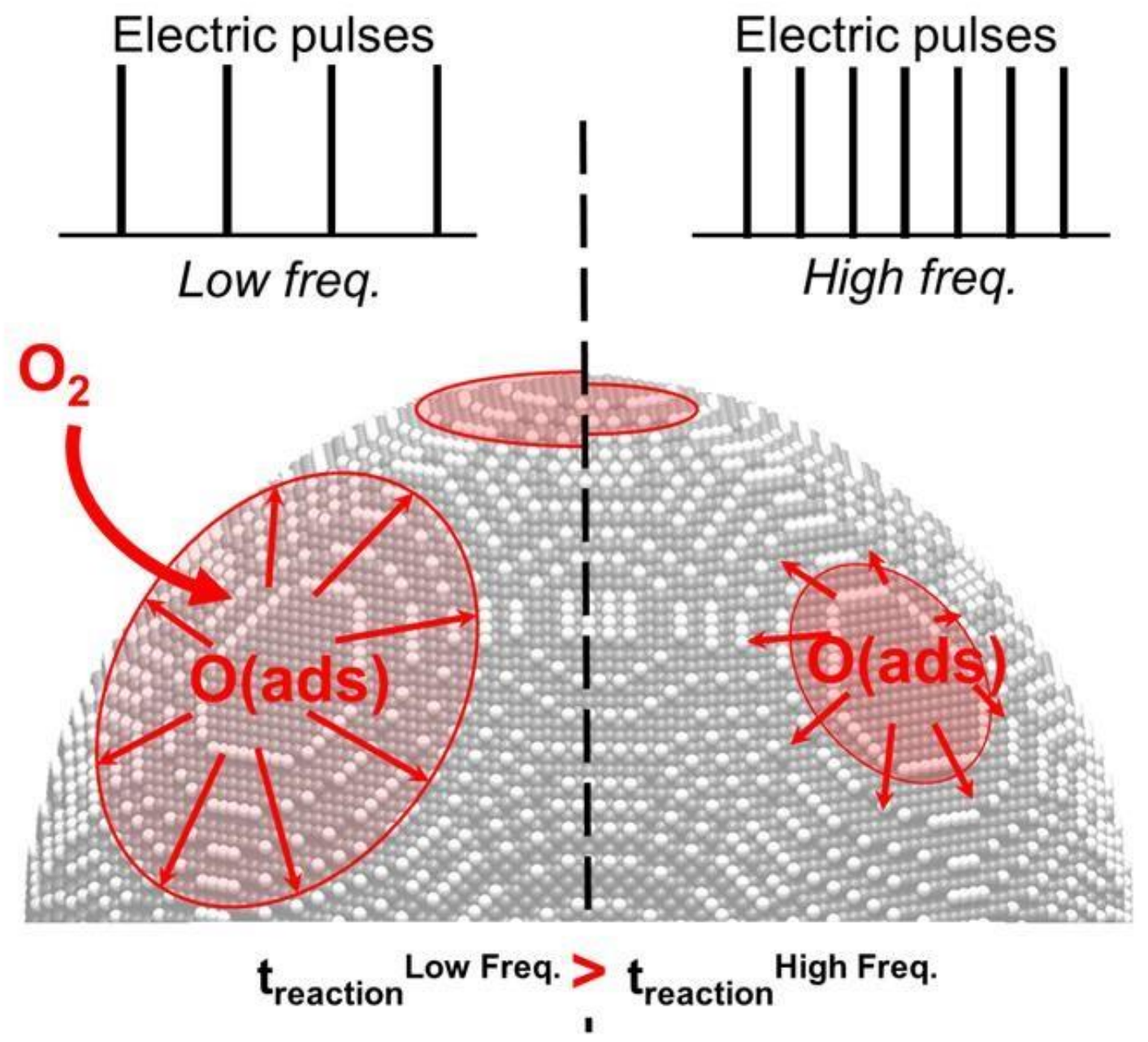

Figure 1. Schematic explanation of electric field assisted oxidation of Fe during operando APT

\section{References}

[1] The work presented here was performed at Pacific Northwest National Laboratory (PNNL), which is operated for the U.S. DOE by Battelle Memorial Institute under Contract No. DE-AC05-76RLO1830. A portion of this research was performed using facilities at the Environmental Molecular Sciences Laboratory, a national scientific user facility sponsored by the DOE Office of Biological and Environmental Research and located at PNNL. S.V.L. acknowledges the Linus Pauling Distinguished Postdoctoral Fellowship program at PNNL for financially supporting the Fe studies. D.E.P. acknowledges support from the Laboratory Directed Research and Development (LDRD) program at PNNL. AD acknowledges support from the U.S. Department of Energy, Office of Science, Basic Energy Sciences, Materials Sciences and Engineering Division as a part of the Early Career Research program. 